\title{
Évaluation des clones de manioc à pulpe jaune pour leur teneur en $\beta$-carotène, rendements en tubercules frais et teneur en matière sèche à Ngandajika en République Démocratique du Congo.
}

\author{
D. Mukendi Tshizembe ${ }^{1 *}$, C. Tshimbombo Jadika ${ }^{1}$, T. Tshiamala Ngeleka ${ }^{1}$, A. Kamukenji Nam'a mbaji1, \\ A. Bombani Bongali et S. Beya Mutombo ${ }^{1}$ \\ ${ }^{1}$ Institut National Pour l'Étude et la Recherche Agronomiques (INERA), Centre de Ngandajika \\ *Corresponding author Email : mukendidonat@yahoo.fr
}

Original submitted in on $8^{\text {th }}$ February 2018. Published online at www.m.elewa.org on $30^{\text {th }}$ April 2018 https://dx.doi.org/10.4314/jab.v124i1.11

\section{RÉSUMÉ}

Objectifs : Cette étude avait pour objectif d'identifier les rendements, les taux de matière sèche, les taux d'amidon et les teneurs en caroténoïdes totaux des clones et de les comparer avec ceux des témoins en vue de sélectionner les nouvelles variétés de manioc biofortifiés afin de combattre la carence en vitamine $A$. Méthodologie et résultats : L'évaluation des 80 clones de manioc à pulpe jaune a été effectuée de 2012 à 2017 au centre de recherche de l'INERA Ngandajika. Le dispositif standard et le dispositif des blocs complètement randomisés avec 4 répétions étaient utilisés respectivement pour le $E C$ et le reste des essais du schéma de sélection. Les résultats ont révélé que GKA12/149, GKA12/141 et GKA12/151 étaient significativement supérieurs pour le rendement en tubercules frais et le TCC, et non significative pour la matière sèche avec Mudiela Manyi. Concernant Kindisa, aucune différence significative sur rendement et MS, sauf en EPR, EAR et EUR1. Pour TCC, GKA12/149 est premier, suivi de GKA12/151, de GKA12/141 et de Kindisa.

Conclusion et application des résultats : les clones GKA12/149 et GKA12/151 se sont montrés, d'une manière générale, plus performants que le témoin amélioré Kindisa et peuvent être proposés à la diffusion. Les croisements divers entre ces génotypes, qui ont enrichi le germoplasme de manioc à pulpe jaune, entre eux et avec d'autres variétés devront se poursuivre.

Mots clés : Manioc bio-fortifié, variétés, sélection

Evaluation of yellow fleshed cassava genotypes for $\beta$-carotene, fresh root tuber yield and dry matter content in Ngandajika, DRC

ABSTRACT

Objectives: The objective of this study was to identify yields, dry matter levels, starch levels, and total carotenoid contents of clones and to compare them with controls to select new bio-fortified cassava varieties to fight vitamin A deficiency.

Methodology and results: The evaluation of the 80 yellow pulp cassava clones was carried out from 2012 to 2017 at the INERA Ngandajika research center. The standard device and the device of the completely randomized blocks with 4 repetitions were used respectively for the clonal evaluation trial (CET) and the rest of the tests of the selection scheme. The results revealed that GKA12/149, GKA12/141 and GKA12/151 were 
significantly higher for fresh tuber yield and Total Carotenoid Content, and not significant for dry matter with Mudiela Manyi. Regarding Kindisa, no significant difference for yield and dry matter content, except in Preliminary Yield Trial (PYT), Advanced Yield Trial (AYT) and Uniform Yield Trial (UYT1). For TCC, GKA12/149 is first, followed by GKA12/151, GKA12/141 and Kindisa.

Conclusion and application: clones GKA12/149 and GKA12/151 were generally more efficient than the improved Kindisa control and can be proposed for diffusion. The various crosses between these genotypes, which have enriched the germplasm of yellow-fleshed cassava with each other and with other varieties, will have to be continued.

Key words: Bio-fortified cassava, varieties, selection

\section{INTRODUCTION}

Quelques 190 millions d'enfants d'âge préscolaire souffrent d'une carence en vitamine $A$, principalement en Afrique et en Asie du Sud-est. Chez les nourrissons et les enfants, la vitamine A est indispensable pour une croissance rapide et pour combattre les infections. Un apport insuffisant de vitamine $A$ peut entrainer une carence provoquant une cécité nocturne et accroître le risque de morbidité et de mortalité consécutives à des infections de l'enfant comme la rougeole et les maladies diarrhéiques (Meda et al., 2000, OMS, 2011). La carence en vitamine $A$ constitue un problème majeur de santé public parmi les groupes vulnérables dont les enfants et les femmes enceintes dans les pays pauvres à faible revenue (Elise, 2013). En République Démocratique du Congo, la carence en vitamine $A$ représente un problème de santé publique (UNICEF 2002, PRONANUT, 2015). Le taux des personnes carencées en Vitamine $A$ en R.D.C. est de $61 \%$ chez les enfants de moins de 3 ans avec un taux de rétinol sérique < à $20 \%$ (PRONANUT, 2015). Pour remédier à cette situation, diverses stratégies de lutte contre la carence en vitamine $A$ ont été entreprises parmi lesquelles la promotion de la production d'aliments riches en vitamine $A$. La biofortification est une nouvelle approche qui consiste à augmenter la teneur en micronutriments directement dans les cultures de subsistance dont se nourrissent les populations pauvres. La biofortification en améliorant le contenu nutritionnel des aliments de base dont se nourrissent déjà les populations, peut devenir une méthode durable permettant d'atténuer la malnutrition en utilisant des aliments bien connus (CIAT/ HarvestPlus, 2009). Améliorer la production du manioc riche en vitamine $A$ est une des stratégies au potentiel fort intéressant étant donné le potentiel productif du manioc par rapport aux autres cultures et ainsi qu'en sa qualité de nourriture de base pour plus de 20 millions des Congolais (CIAT/HarvestPlus 2009, Ross, 2016). Les études récentes ont démontré que la consommation du manioc biofortifié riche en provitamine $A$, peut être une nouvelle approche efficace pour améliorer le statut de vitamine $A$ et que la consommation de cette denrée alimentaire apporte des gains modestes de la concentration en sérum rétinol et une grande augmentation de la concentration en $\beta$-carotène (Michael et al. 2013, Elyse et al., 2016). 

carotène, rendements en tubercules frais et teneur en matière sèche à Ngandajika en RDC

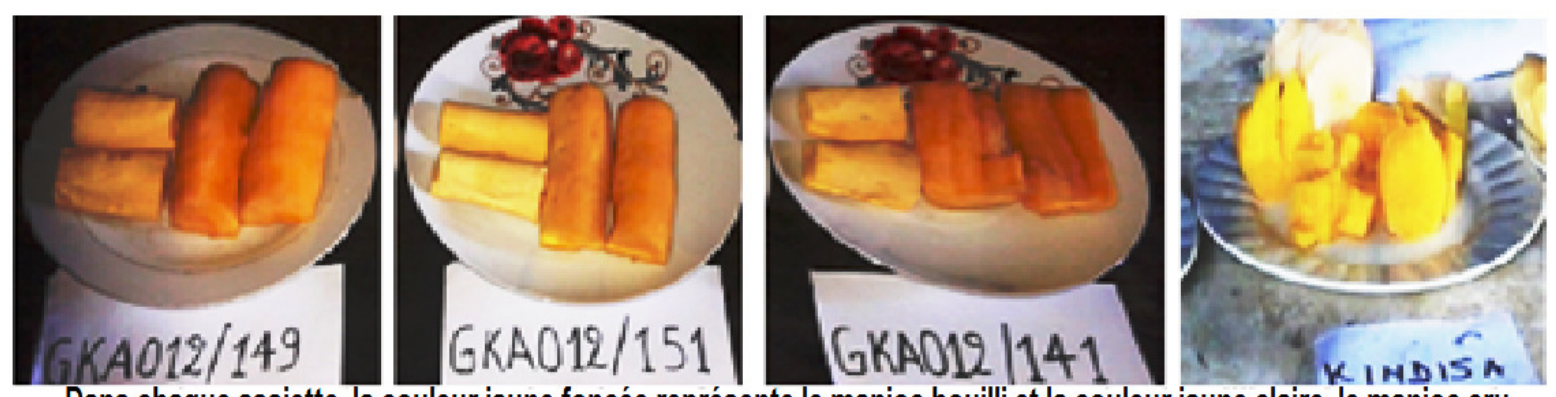

Dans chaque assiette, la couleur jaune foncée représente le manioc bouilli et la couleur jaune claire, le manioc cru

Cependant les tubercules des clones riches en caroténoïdes ont une faible teneur en Matière Sèche et des propriétés culinaires médiocres (Moorthly et al, 1990 cité par Njenga et al. 2014). Tel est le cas de la variété Kindisa développée à Mvuazi, et qui est l'unique variété riche en beta carotène diffusée en $\mathrm{RDC}$ jusqu'à ce jour. Cette variété quoique riche en provitamine $A$ et très productive, a une faible teneur en matière sèche, ne se ramolli pas à la cuisson et exige un temps de séchage très long, ce qui réduit son acceptabilité par les consommateurs du Kasaï. Pour cette raison, il est indispensable de diversifier les variétés de manioc biofortifiées étant donné que la réponse des génotypes à l'environnement et les critères de choix pour l'adoption par les consommateurs diffèrent d'un lieu à l'autre. La biofortification du manioc peut être réalisée en utilisant les techniques conventionnelles d'amélioration en utilisant les génotypes existants

\section{MATERIEL ET METHODE}

Site d'étude: L'étude s'est déroulée au centre de recherche de l'INERA Ngandajika $\left(23^{\circ} 57^{\prime} \mathrm{E}, 06^{\circ} 48^{\prime} \mathrm{S}\right.$ et $754 \mathrm{~m}$ d'altitude) durant sept années agricoles, de 2012 à 2017. Le climat du milieu est du type Aw4 suivant la classification climatique de Köppen, caractérisé par l'alternance de deux saisons (la saison pluvieuse et la saison sèche). La répartition annuelle de pluie est bimodale avec les pics en janvier et en Avril. La pluviométrie annuelle varie de 1400 à $1500 \mathrm{~mm}$ de pluie, la température annuelle moyenne est d'environ $24^{\circ}$. Selon les informations tirées de l'IRAZ, 1989, la texture du sol est sablo-argileuse à argile lourd, de coloration rouge à ocre rouge, et de bonne structure. La fraction argileuse est peu importante et varie selon les localités. Le pH du sol varie de 5.2 à 6.8 .

Méthode : Le Programme National Manioc (PRONAM), antenne de Ngandajika a réalisé ces travaux de
(Jenning et al., 1999, Darwin Oet al., 2011, Howarth et al., 2013, Njenga et al., 2014). En vue de développer les génotypes riches en provitamine $A$, productifs, résistants aux ennemis de la culture et ayant des qualités organoleptiques appréciées par les consommateurs, cette méthode a été utilisé à Ngandajika, par HarvestPlus et l'Institut National pour la Recherche Agronomique (INERA). L'hypothèse de ce travail est que les croisements contrôlés et non contrôlés des meilleures génotypes à pulpe jaune et à pulpe blanche existants à la station pourraient conduire à obtenir les clones ayant des bonnes teneurs en $\beta$-carotène et des qualités organoleptiques acceptables par les consommateurs. II s'avère aussi nécessaire de diversifier la gamme des variétés de manioc biofortifiés adaptées aux différentes écologies de nos provinces.

recherche, en utilisant la méthode conventionnelle. Celleci consiste à installer chaque année un bloc de croisement comprenant les meilleures variétés à pulpe jaune qui sont croisées entre elles. Les graines obtenues de croisements contrôlés et non contrôlés sont évaluées dans la Pépinières à Graines (PG). Les meilleurs sauvageons sélectionnés dans la PG sont criblés dans l'Essai Clonal $(E C)$ pour la résistance aux ennemis de la culture, la teneur en $\beta$-carotène ainsi que la morphologie de la plante. Les génotypes sélectionnés dans l'EC sont évalués dans l'Essai Préliminaire de Rendement (EPR) où le rendement de chaque clone est mesuré en plus de traits observés au niveau de l'EC. Ce criblage se poursuit ainsi en Essai Avancé de rendement (EAR) et en Essai Uniforme de Rendement (EUR) respectivement à la cinquième et la sixième année (PRONAM Ngandajika, 2017). 

carotène, rendements en tubercules frais et teneur en matière sèche à Ngandajika en RDC

Matériel Végétal : Les croisements contrôlés et ceux à pollinisation ouverte effectués dans le bloc de croisement en 2011 entre 17 familles avaient permis d'obtenir 3000 graines. Le criblage des 2873 sauvageons issus de ces graines dans la PG 2012 avait conduit à identifier 80 génotypes à pulpe jaunes dont 30 Full-Sib et 50 Half-sib qui furent installés dans l'EC 2013. A l'issu de l'évaluation à ce stade de sélection, 4 clones issus de 3 familles furent sélectionnés selon les détails repris dans le tableau1.

Tableau 1 : Clones sélectionnés pour l'EPR jaune 2014, TCC obtenu à l'EC

\begin{tabular}{lll}
\hline Clones & Croisement & TCC $(\mu \mathrm{g} / \mathbf{g})$ \\
\hline GKA2012/149 & Kindisa x Mudiela Manyi & 10,95 \\
GKA2012/141 & TME 419 x Tshilobo & 9,2 \\
GKA2012/151 & Kindisa x Mudiela Manyi & 12,44 \\
GKA2012/150 & Kindisa x Mudiela Manyi & 19,5 \\
\hline
\end{tabular}

Le clone GKA2012/150 fut aussi éliminé au stade de l'EPR à cause de l'apparition des symptômes de la Mosaïque africaine du manioc. Les 3 clones restants ont ainsi terminé le cycle de sélection suivant le schéma standard de sélection du manioc en EAR, EUR1 et EUR2. Dispositifs Expérimental: L'EC 2013 était installé suivant le dispositif standard (check plot design) où chaque clone occupait une parcelle d'une ligne de 5 pieds. Après chaque série de 10 clones suivaient le témoin amélioré (Kindisa) et le témoin local (Mudiela Manyi). Le dispositif des blocs complètement randomisés avec 4 répétitions, où chaque clone occupait une parcelle élémentaire d'une ligne de 10 pieds était utilisé pour l'EPR 2014. Ce même dispositif était utilisé pour l'EAR2015, pour l'EUR12016 et pour l'EUR2 2017, mais pour ces trois essais, la parcelle élémentaire comprenait 4 lignes de 10 pieds. Les écartements adoptés étaient de $1 \mathrm{~m} \times 1 \mathrm{~m}$. Le nombre des clones évalués dans ces essais a varié comme suit : EC $2013: 80$ clones, L'EPR $2014: 4$ clones dont GKA2012/149, GKA2012/141, GKA2012/151 et GAK2012/150. Les 2 témoins utilisés étaient : Kindisa (témoin amélioré) et Mudiela Manyi (témoin local). De l'EAR à l'EUR2: 3 clones dont: GKA2012/149, GKA2012/141, GKA2012/151 étaient mis en compétition avec les mêmes témoins.

Collecte des données : Le criblage dans l'Essai Clonal a porté sur la résistance aux maladies et ennemis de la culture, la morphologie de la plante, la forme et le poids des tubercules frais et la teneur en béta carotène. Les données étaient prises sur les 3 pieds internes. Dans l'EPR 2014 les observations étaient faites sur les 8 plants internes, tandis que 16 pieds internes étaient observés dans les 3 autres essais (EAR, EUR1, EUR2). Les données sur la croissance et les attaques des maladies et ravageurs de manioc étaient prises trimestriellement dans chaque essai. Les données prises à la récolte étaient, le Nombre de Plant Récolté (NPR), le Nombre de Tubercules Frais Commercialisables (NTFC), le Nombre de Tubercules Frais Non Commercialisables, Le Nombre des Tubercules Pourries (NTP), le poids des Tubercules Frais Commercialisables (PTFC), le poids des tubercules frais Non Commercialisables (PTFNC), la biomasse, le poids dans l'air et le poids dans l'eau des tubercules frais. L'estimation de la teneur en matière sèche et en amidon des variétés en évaluation était faite suivant la méthode de gravité spécifique. Cette méthode consiste à prélever et peser simultanément dans l'eau $(\mathrm{Pe})$ et à l'air libre $(\mathrm{Pa})$, pour chaque clone, un échantillon de racines tubéreuses fraiches dont le poids varie entre 300 et 500 g. La gravité spécifique calculée comme suit, $X=\mathrm{Pa} /(\mathrm{Pa}$ $-\mathrm{Pe}$ ), est introduite dans les équations respectives pour donner les teneurs en matière sèche et amidon (W.M.G Fukuda et al., 1998) Matière sèche $(\%)=158.3 * X-142$. Le test iCheck carotène était utilisé pour l'analyse des caroténoïdes dans le manioc. L'analyse des caroténoïdes était faite en suivant le mode d'emploi iChek carotène (www.icheckAcademy.org, 2016, IITA, 2012)

Analyse Statistique : L'analyse statistique des données enregistrées dans l'essai clonal était effectuée à l'aide d'Excel suivant la méthode de Klaus Rohrmoser et Manfred Wermke pour l'analyse d'un essai installé suivant le Check plot design (Klaus R. et Manfred W., 1986). L'analyse statistique des données collectées dans l'EPR, l'EAR, l'EUR1 et l'EUR a été effectuée par le biais du test d'analyse de variance (ANOVA) à l'aide du logiciel Statistix 8.0. La mise en évidence des différences significatives entre les traitements a été réalisée au moyen du test LSD au seuil de $5 \%$ de probabilité. 
Mukendi et al., J. Appl. Biosci. 2018 Évaluation des clones de manioc à pulpe jaune pour leur teneur en $\beta$ carotène, rendements en tubercules frais et teneur en matière sèche à Ngandajika en RDC

\section{RESULTATS}

Les résultats obtenus durant les 4 années d'évaluation pour le rendement en tubercules frais, la teneur en bétacarotène, la teneur en matière sèche et la teneur en

amidon sont présentés respectivement dans les figures : $1,2,3$ et 4.

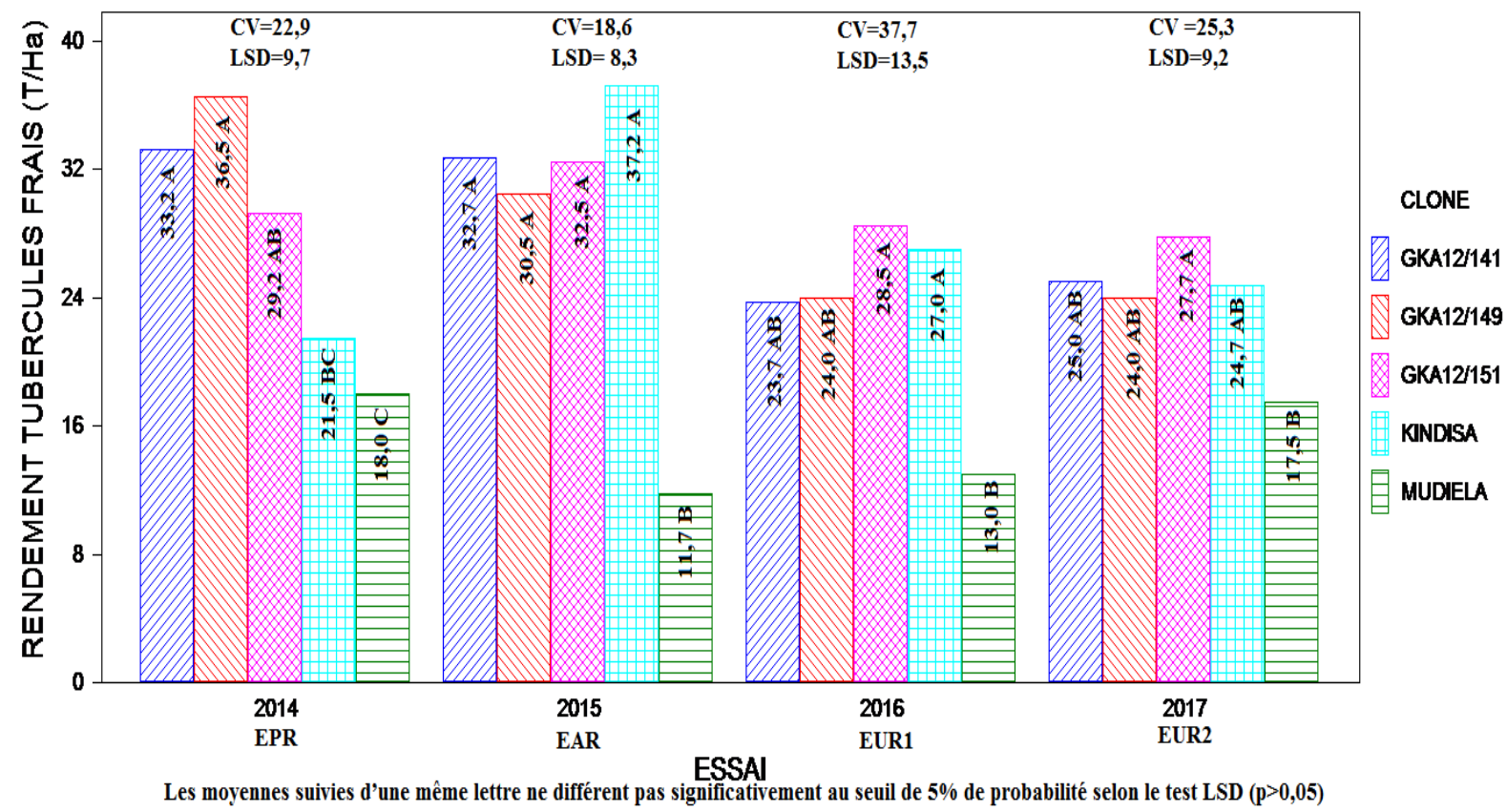

Figure1 : Les rendements successifs en tubercules frais de clones durant les 4 ans de selection de l'EPR à l'EUR2 $(\mathrm{T} / \mathrm{Ha})$

De la lecture des résultats sur le rendement en tubercule frais consignés dans la figure1, on note que les 3 clones biofortifiés en évaluation GKA12/149, GKA12/141 et GKA12/151 ont eu des rendements en tubercules frais significativement supérieurs au témoin local Mudiela Manyi, sauf en EUR2 où GKA12/151 seul a montré une différence significative. Par rapport au témoin amélioré Kindisa, qui est naturellement très productif, il n'y a pas eu de différence significative de l'EAR jusqu'à l'EUR2. Au niveau de l'EPR, les clones GKA12/141 et GKA12/149 avaient obtenu des rendements significativement supérieurs à celui de ce témoin. 


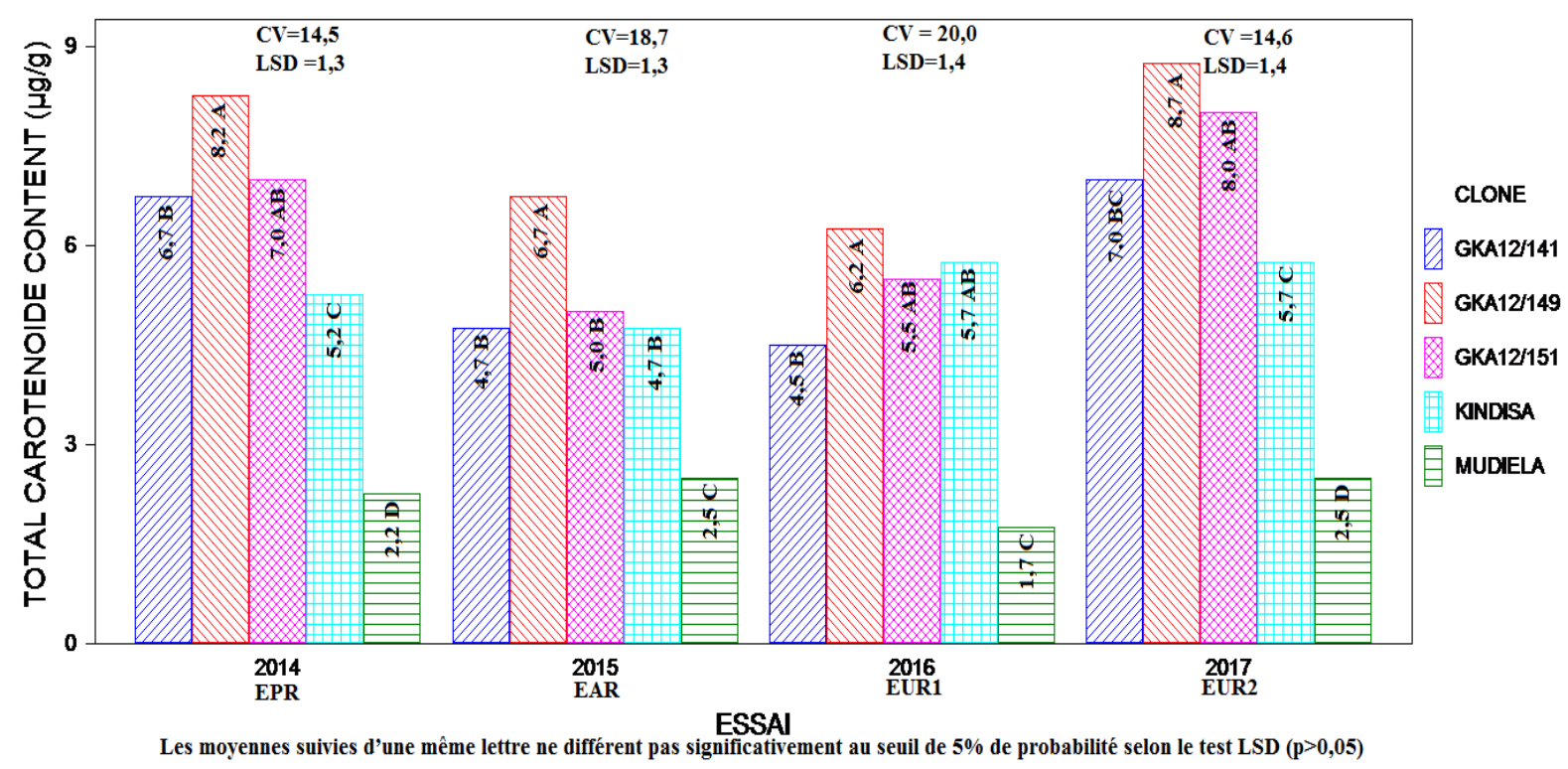

Figure2 : Les TCC successifs de clones durant les 4 ans de selection de l'EPR à l'EUR2 ( $\left.\mu \mathrm{gg}^{-1}\right)$

Les résultats sur la teneur en béta carotène repris dans le tableau 2 indiquent que tout au long de cette évaluation, excepté en EUR1, le clone GKA12/149 avec respectivement $8,2 \boldsymbol{\mu g g}^{-1}, 6,7 \boldsymbol{\mu g g}^{-1}, 6,2 \boldsymbol{\mu g g}^{-1}$ et 8,7 $\mu \mathrm{gg}^{-1} \mathrm{~s}^{\prime}$ est montré plus performant que le témoin amélioré Kindisa. Sa TCC était significativement supérieure à celle de Kindisa au niveau de l'EPR, de l'EAR et de l'EUR2. Au niveau de l'EUR1, il n'y a pas eu de différence significative mais, il lui était numériquement supérieur. Le clone GKA12/151 a manifesté aussi des TCC significativement supérieurs à celles de Kindisa au niveau de l'EPR et de l'EUR2 et des TCC sans différence significative au niveau de l'EAR et de l'EUR1. Le clone GKA12/141 n'était pas significativement différent de Kindisa dans tous les essais. Par rapport au témoin local Mudiela Manyi, tous les clones en évaluations étaient significativement supérieurs.

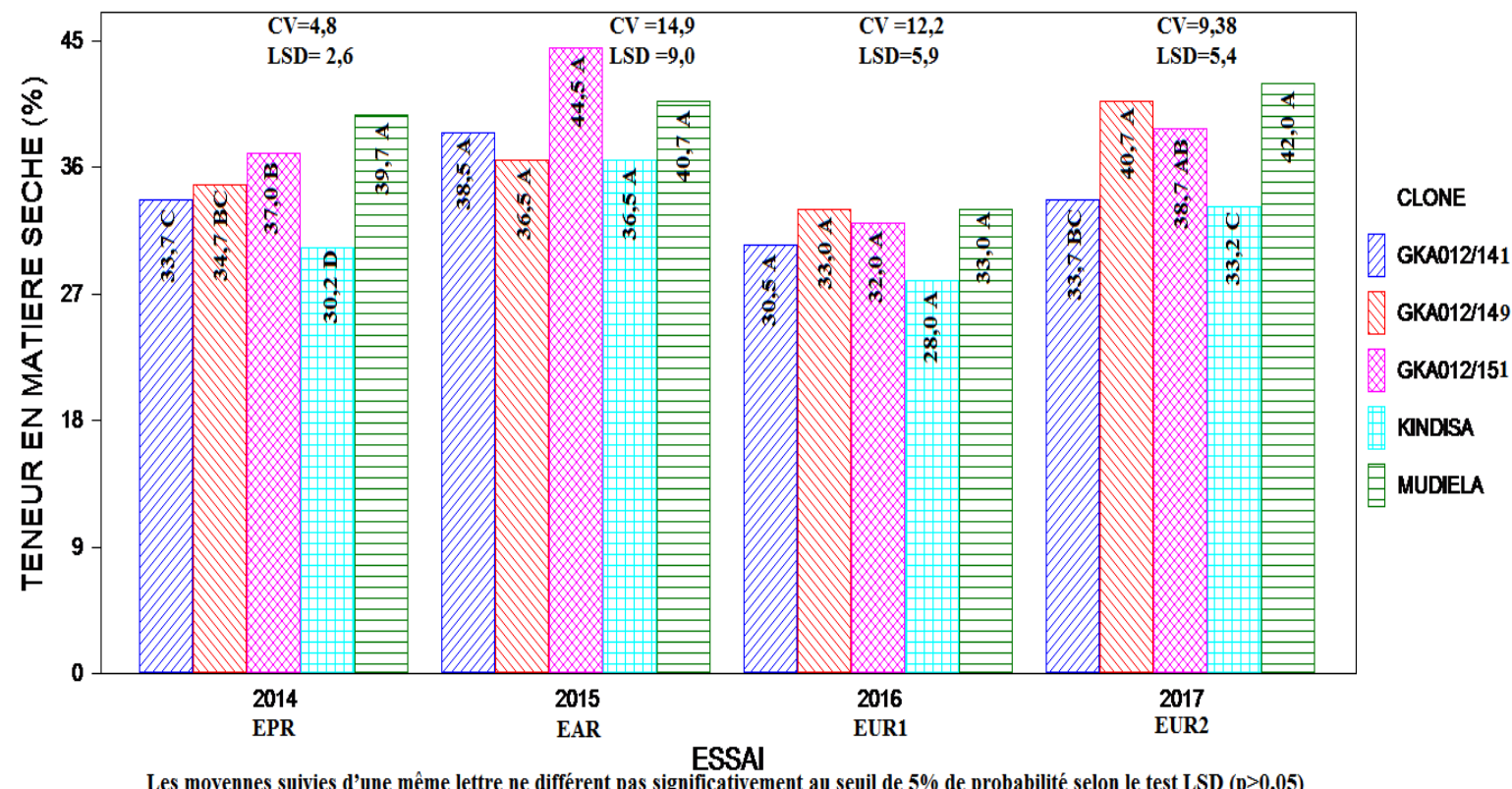

Figure3 : Les teneurs en matière séche successifs de clones durant les 4 ans de selection de l'EPR à l'EUR2 (T/Ha) 

carotène, rendements en tubercules frais et teneur en matière sèche à Ngandajika en RDC

En ce qui concerne le taux de la matière sèche, les résultats présentés dans le tableau 3 montrent que le témoin local Mudiela Manyi, qui est réputé pour une meilleure teneur en matière séche, n'a pas dépassé significativement les clones GKA12/149 et GKA12/151, sauf à l'EPR.
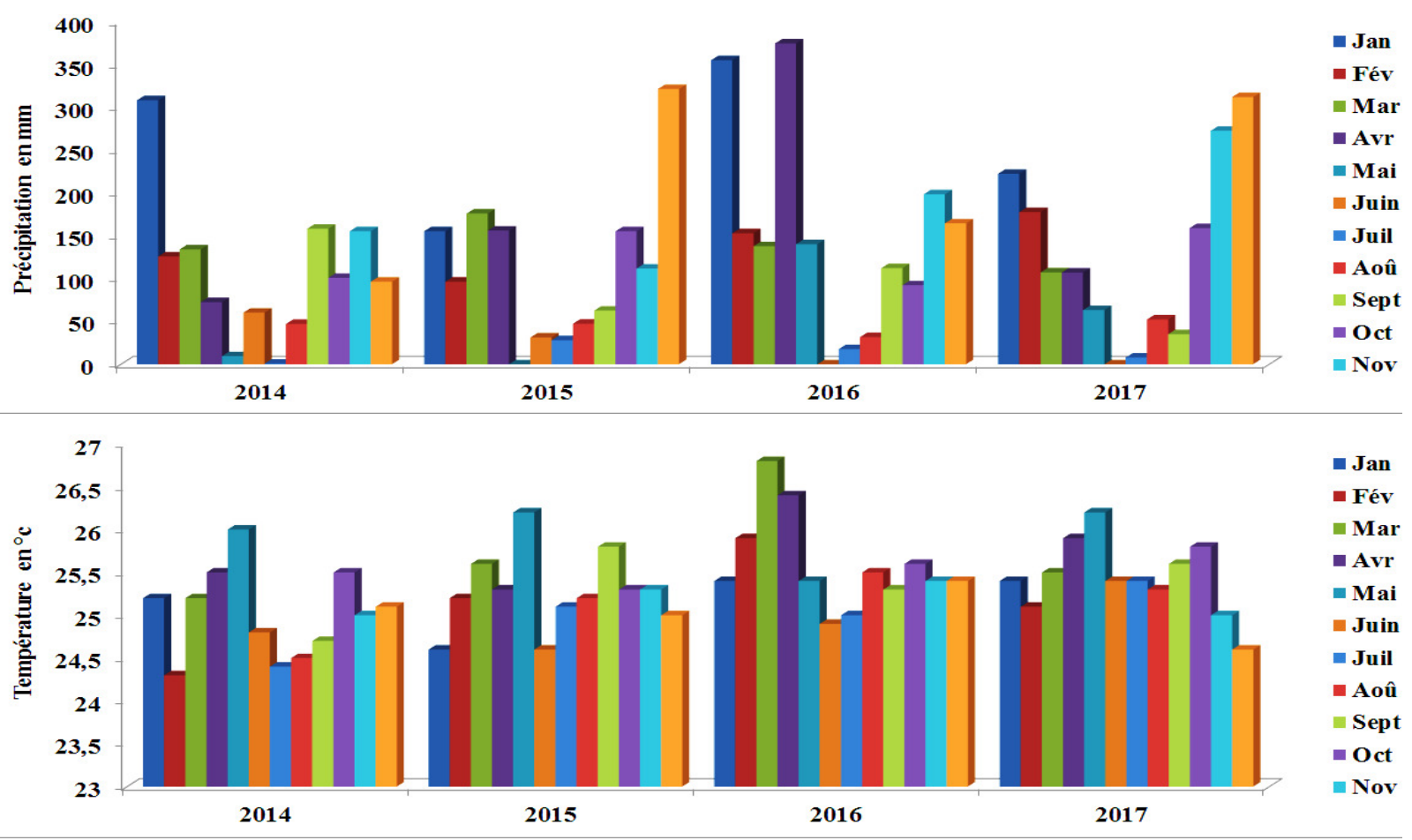

Source : Station météorologique INERA Ngandajika

Figure 4 : variation de la pluviométrie et des températures de 2014 à 2017 durant la période de sélection

Les données climatiques que révèle la figure 4 montrent comment les quantités des pluies et les températures mensuelles ont varié chaque année, durant le cycle de

\section{DISCUSSION}

D'après nos résultats, le criblage de 80 clones à pulpe jaune a permis de sélectionner 4 clones performants pour les principaux critères/traits de sélection de manioc bio fortifié pris en compte.

Rendement en racines Tubéreuses Fraiches: En considérant les rendements en tubercules frais des clones GKA12/141, GKA12/149 et GKA12/151, par rapport à ceux du témoin local Mudiela Manyi, à travers tout le cycle de sélection, on note que le rendement minimum de 23,7 t/ha obtenu par le clones GKA12/141 et le rendement maximum de 36,5 tha obtenu par GKA12/149 sont de loin supérieur au rendement maximum de 18 tha enregistrés chez ce témoin local. Comparés au témoin amélioré Kindisa dont le rendement a varié de 24,7 t/ha ( $\mathrm{min})$ à 37,2 t/ha (max), on constate sélection. Elles ont aussi entrainé la variation des conditions environnementales dans lesquelles chaque essai a été conduit.

qu'il n'y a presque pas de différence et que les rendements de ces clones sont bons étant-donné la grande valeur de Kindisa en terme de rendement en racines tubéreuses. Le fait que la variété locale est peu productive par rapport aux variétés améliorées est en accord avec les conclusions de plusieurs travaux dont les résultats ont démontré la supériorité des variétés améliorées sur les variétés locales (Muimba $\mathrm{K}$ et al., 1986, N'zué et al., 2004, Adekambi et al., 2010, Bakayoko S. et al., 2012). L'utilisation de variétés améliorés telle que Kindisa et TME419 comme parents a permis d'obtenir des clones ayant aussi des rendements en tubercules frais intéressants. Ceci corrobore le constat fait par certains auteurs selon lequel, la sélection récurrente pratiquée à l'IITA, consiste à transmettre dans 
les nouvelles variétés des caractères d'intérêt agronomique provenant de géniteurs préalablement choisis (Bakayoko et al., 2012). La variation des rendements de tous les clones chaque année dans les essais pendant ce criblage est liée au changement des sites et à la variation des conditions climatiques Chaque année au cours de ce travail comme décrit par la figure 4 . Beaucoup des travaux ont déjà mis en évidence le fait que la performance des variétés varie significativement en fonction des génotypes et de l'environnement (E.S. Bah et al., 2010, N. P. Ntawuruhunga et al., 2010, N. G. Maroya et al., 2012).

Teneur en beta carotene: L'analyse des teneurs en caroténoïde total a montré des différences significatives entre les variétés. Ceci est en accord avec les travaux de G.O. Agbaye et al., 2007 qui ont trouvé des différences significatives entre les TCC des différents clones. Les TCC du témoin local : 1,5 à 2,5 $\mu \mathrm{g} / \mathrm{g}$ étaient inférieures à celles de ces nouvelles variétés et celles du témoin amélioré Kindisa 4,7 $\mu \mathrm{g} / \mathrm{g}$ à $5,7 \mu \mathrm{g} / \mathrm{g}$ étaient soit inférieures ou égales à celles de ces nouvelles variétés. Ces résultats sont en accords avec ceux des travaux qui ont démontré une haute héritabilité du caractère beta carotène et la possibilité de biofortifier les variétés existantes par la sélection conventionnelle récurrente (D. Ortiz et al., 2010, Njenga P. et al., 2014). Le clone GKA149 occupe la première place pour le TCC $\left(6,2 \mu \mathrm{ug}^{-1}\right.$ à $\left.8,7 \mu \mathrm{\mu gg}^{-1}\right)$ suivi de GKA12/151(5,0 $\mu g^{-1}$ à $\left.8,0 \mu g^{-1}\right)$, de GKA12/141(4,5 $\mu g^{-1}$ à $\left.7 \mu g g^{-1}\right)$, de Kindisa $\left(4,7 \mu g^{-1}\right.$ à $\left.5,7 \mu \mathrm{gg}^{-1}\right)$ et Mudiela Manyi $\left(1,5 \mu \mathrm{gg}^{-1}\right.$ à $\left.2,5 \mu \mathrm{\mu g}^{-1}\right)$. Ceci indique que la valeur alimentaire de témoins est inférieure ou égale à celle des nouvelles variétés qui peuvent à cause de cela être mise à la disposition des

\section{CONCLUSION}

En mettant en compétition les différents clones de manioc à pulpe jaune avec les témoins, on s'était assigné comme objectif, de déterminer et de comparer leurs performances afin de sélectionner et de diversifier les meilleures variétés biofortifiées à proposer à la diffusion en vue de lutter contre les carences en vitamine A. Au terme de ce travail, il s'est dégagé que les clones GKA12/149 et GKA12/150 se sont montrés, d'une

\section{REMERCIEMENT}

Nous remercions les autorités du projet HarvestPlus et de I'INERA pour leurs soutiens matériel et financier tout au long de ce travail. Nos remerciements particuliers s'adressent au Dr Anderson Meike et Ir Msc. Sylvain consommateurs bien que le seuil de $15 \mu \mathrm{gg}^{-1}$ fixé par HarvestPlus ne soit pas encore atteint.

Teneur en Matière Sèche (MS) : Les taux de matière sèche et d'amidon enregistrés à travers ce cycle de sélection ont varié chez toutes les variétés chaque année. Ce changement serait dû à la variation des conditions climatique chaque année et au changement de sites d'études. Van Oirschol et al., 2000 cité par D.Ortiz et al., 2010 avaient montré que les conditions environnementales et les partiques culturales peuvent avoir un impact important sur le changement de la teneur en matière sèche. N.G. Maroya et al., 2012 avaient aussi rapporté que les variétés de manioc souvent démontrent une adaptation spécifique due à leur sensibilité élevée aux interactions génotype $x$ environnement ( $G \times E$ ) aussi bien que pour les performances à court termes et à long termes des clones dans les essais. Les taux de matière sèche enregistrés chez la variété GKA12/151 (32 à $44,5 \%$ ) et la variété GKA12/149 (33 à 40,7\%) se rapprochent de ceux observés chez le témoin local Mudiela Manyi (33 à 42,0\%) qui est apprécié localement pour ce paramètre. Ces 2 clones étant issus des croisements entre Kindisa (faible teneur en MS) et Mudiela Manyi (teneur élevée en MS), il est intéressant de noter qu'il est possible d'améliorer la teneur en MS des variétés à faible teneur par le croisement avec celle à teneur élevée. L'héritabilité élevée de ces paramètres a été démontrée par les travaux de $P$. Ntawuruhunga et al., 2010. Le clone GKA12/141 (30,5 à 38,5 \%) s'est comporté de façon presque identique à Kindisa (28 à $36,5 \%$ ). Cette tendance était observée également pour le taux en amidon.

manière générale, plus performants que le témoin amélioré Kindisa et peuvent être proposés à la diffusion. Le clone GKA012/141, qui lui est égale, est aussi intéressant. Les croisements divers entre ces génotypes, qui ont enrichi le germoplasme de manioc à pulpe jaune entre eux et avec d'autres variétés devront se poursuivre en vue d'atteindre le seuil de $15 \mu \mathrm{g} / \mathrm{g}$.

Bidiaka de HarvestPlus pour le choix porté sur notre Centre de recherche dans l'exécution de ce Projet. Que nos collègues de PRONAM Ngandajika trouvent aussi à travers ces lignes notre gratitude pour le travail abattu. 


\section{REFERENCES BIBLIOGRAPHIQUES}

Bakayoko S., Kouadio K.K.H, Soro D. Tschannen A., Nindjin C. Dao D., Girardin O., Rendements en tubercules frais et teneurs en matière sèche de soixante-dix nouvelles variétés de manioc (manihot esculenta Crantz) cultivées dans le centre de la Côte-d'Ivoire, journal of animal and plant sciences, 2012. Vol.14.

CIAT/HarvestPlus, 2009. Améliorer les cultures pour une bonne alimentation, note d'information, www. HarvestPlus.org.

Darwin Ortiz,Teresa Sanchez, Nelson Morante, Hernan Ceballos, Helena Pachon, Myriam c. Duque, Alba Lucia Chàvez and Andrés Felipe E. 2011, Sampling Strategies for proper quantification of carotenoid content in cassava breeding, in journal of plant breeding and crop science vol.3(1),pp. 14-23.

Elyse F.T, 2013. Yellow cassava: efficacy of provitamin A rich cassava on improvement of vitamin A status in Kenyan school children, hoostdstukintroductie thesis.

Elyse F Talsma, Inge D Brouwer, Hans V., Gloria NK.M., Alice M.M., Ayse Y.D., Busie M. D., Erick B., and Michael BZ.and Alida M-B., 2016 : Biofortified yellows cassava and vitamin $A$ status of Kenyans children : a randomized controlled trial, the American journal of clinical nutrition.

Frangoie Ngoie, 2015. Rapport de la mission de caractérisation effectuée à Mvuazi, IITA /DRC.

Howarth Bouis, JanLow, Margaret McEwan, Sherry Tanumihardjo, 2013. Biofortification: Evidence and lessons learned linking agriculture and nutrition.

IRAZ (Institut de Recherche Agronomique et Zootechnique de la CEPGL), 1989. Étude pédologique de huit sites repères pour les essais au sein de la CEPGL (Moso, Mashitsi, Rubona, Karama, Yangambi, Mulungu, Gandajika, M'vuazi).

Klaus R. et Manfred W., manuel des essais au champ, CTA, eschborn, 1986

M. NaitormbaideM. , Djondang K, Mbayoudel $\mathrm{K}$ et Nekouam N, 2009: Sélection et transfert des variétés de manioc tolérantes à la sécheresse et à la mosaïque africaine, rapport final du projet d'appui aux services agricoles et aux organisations des producteurs, Tchad.

Michael R. LF., Leslie R. W. , Dustin J.B and Betty J.B., 2013: Biofortified cassava increases $\beta$-carotene and vitamin $A$ concentrations in the TAG-rich plasma layer of Americans women, British Journal of Nutrion.

Njenga Peninah, Edema Richard and Kamau Joseph, 2014. Combining ability for beta-carotene and important quantitative traits in a cassava $\mathrm{f1}$ population, journal of plant Breeding and Crop Science.

N.G. Maroya, Peter Kulakow, Alfred G.O. Dixon, Busie B. Maziya-Dixon, 2012: Genotype x Environment Interaction of Mosaic Disease, Root Yields and Total Carotene Concentration of Yellow-Fleshed Cassava in Nigeria.

P. Ntawuruhunga and A.G.O. Dixon, 2010 : quantitative variation and interrelationship between factors influencing cassava yield, Journal of Applied Biosciences 26: 1594-1602, ISSN 1997-5902,

N. Meda, P. Chevalier, C. Mathieu-Daude, 2000. Manifestations oculaires liées à la carence en vitamine $\mathrm{A}$ en zone rurale du Burkina Faso, Médecine Tropicale, vol $60 \mathrm{~N}^{\circ} 1$.

OMS, 2011. Supplémentassions en vitamine A pour les nourrissons et les enfants de 6 à 59 mois, Documents OMS, ISBN9789242501766.

OMS, 2011. Directive : supplémentassions en vitamine $A$ chez les nourrissons et les -enfants de 6-59 mois. Genève.

PRONANUT, 2015. Carence en micronutriments, in PNDS VOLET NUTRITION 2011-2015.

PRONAM Ngandajika, rapport annuel des activités, 2017.

Ross M. Welch, 2002. Breeding Strategies for biofortified staple foods to reduce Micronutrient Malnutrition Globally, journal of Nutrition 132: 495S-499S.

M.L. Rutikanga, D.S.O. Osiru, J.K. Makelele et C. Bayituliki, 2004. Effects of location and age at harvest on the yield of cassava genotypes (Manihot esculenta Crantz) in Ruanda. Proceedings: 9th ISTRC-AB Symposium, Kenya, page 507.

UNICEF, WHO, 200. Global Database on vitamin A deficiency, in vitamin and mineral Nutrition Information system (VMNIS).

www.icheckAcademy.org: I check Carotènes: Analyse des caroténoïdes dans le manioc : étape-parétape, 2016.

W. M. G. Fukuda, C. L. Guevara, M. E. Ferguson and R. Kawuki. 1998. Descriptors for cassava Morphological Characterisation (EMBRAPACNPMF, Documentos, 78). 